\title{
RELACIÓN ENTRE LOS COMPLEJOS MEDIOS-FIN CON LOS TIPOS DE AFRONTE ANTE LA CRISIS ECONÓMICA
}

\author{
Luis A. Vicuña Peri \\ Mildred Paredes Tarazona \\ Hector Manuel Hemandez Valz. \\ Rolando S. Solis Narro
}

\begin{abstract}
RESUMEN
La presente investigación tiene como intención conocer la relación existente entre el complejo medios-fin (Adler A.) con los tipos de afronte ante la crisis económica, estudiada desde la perspectiva de la teoría de las atribuciones según Peterson y Villano va. Queremos explorar si la orientación productiva de la personalidad se relaciona con el estilo atribucional optimista. Así mismo hemos analizado el papel que juega la condición laboral y el género en la mencionada correlación.

Hemos trabajado con una muestra aleatoria constituida por 483 sujetos de Lima, representativa al 0.05 de margen de error, a quienes se les aplicó dos cuestionarios válidos y confiables, el primero fue el Monofactorial de Estilos de Vida y el segundo fue el ASQ-CIDE, de estilos atribucionales. Efectuado el análisis se concluye que hay correlación inversa negativa entre la personalidad productiva con el estilo atribucional pesimista, así mismo en el grupo de empleados las correlaciones entre la personalidad productiva y el estilo atribucional optimista es significativamente mayor respecto al grupo de desempleados. Por otra parte la contrastación de correlaciones hecha" en función al género nos dice que esta no es una variable que afecte en medida significativa a las correlaciones.

En resumen podemos afirmar que la orientación productiva y el estilo atribucional optimista son característicos en los sujetos con empleo lo que lleva a plantear en próximos estudios si el tipo de personalidad y el estilo atribucional influyen sobre el éxito en conseguir o para generar su propio empleo, pues el presente estudio nos abre la posibilidad correlacional que así es.
\end{abstract}

Palabras clave: Complejo medios-fin, Estilo atribucional, Compuesto negativo, Estilo atribucional pesimista.

\begin{abstract}
The present investigation has as intention to know the existent relationship among the complex means end (Adler,A) with the types of it confronts before the economic crisis, studied from the perspective of the theory of the atributions according to Peterson and Villanova. We want to explore if the productive orientation of the personality is related with the style optimistic atribucional. Likewise we have analyzed the paper that plays the labor condition and the gender in the mentioned correlation.

We have worked with a constituted aleatory sample OR 483 subject of Lima, representative at the 0.05 of error margin to who you/they were applied two worth questionnaires and reliable, the first one was the Monofactorial of lifestyles and the second was the ASQ-CIDE, of styles attributions. Making the analysis you concludes that there is correlation inverse negative among the productive personality with the style optimistic atribucional it is significant bigger regarding the group of unemployed. For another art the contrastación of correlations made in function to the I generate he/she tells us that est year is a variable that affects in significant stocking to the correlations.

In summary we can affirm that the productive orientation and the style optimistic atribucional are characteristic in those subject with employment what takes to outline in next studies if the type of personality and the style atribucional influence about the success in getting or to generate their own employment, beca use the present study doesn't open the possibility correlacional that is this way.
\end{abstract}

Key word: Complex means-end are, Used atribucional, Negative compound, Style pessimistic atribucional. 
La personalidad y los estilos atribucionales inciden en la economía al modificar el comportamiento económico: de la pobreza a la bonanza o a la inversa; observándose que la unidad económica básica, la familia, como las diversas organizaciones como son las empresariales, tanto en do y exogrupales están potencializadas por los recursos de sus miembros, otorgando peculiaridad a cada una de las unidades económicas no sólo por el objetivo que cumplen en el mercado o por la interrelación de ambos actores en forma directa, es decir a un nivel microeconómico, sino por el contexto (medio) que lo constituya la gestión o dirección que enlace y ofrezca las condiciones de expresión o uso de dichas potencialidades.

Es indudable que el comportamiento microeconómico se afecta por la percepción de los agentes económicos tanto como por las ingerencias del agente económico normativo. Este agente normativo orienta la economía del país según el marco del enfoque de política económica y la gestión de los diferentes sectores, comportamiento macro-económico que afecta directamente a los agentes económicos a nivel del individuo. El mismo genera aprendizajes sobre los comportamientos económicos que se ve afectado en sus relaciones personales e interpersonales tanto para su ajuste o enfrentamiento de la realidad económica como parte de su entorno.

Uno de los problemas cruciales de la Psicología gira en torno a la Personalidad, Allport (1965) resume que existen cincuenta definiciones sobre éste proceso mediador, sin que, ninguna logre llenar los vacíos que ellas mismas han generado al enfatizar en alguno de sus componentes. Hecho que no niega la existencia de la personalidad, y la ubicamos como un proceso mediador que organiza y regula a los otros procesos mediadores y variables condicionantes, asumimos que su organización y desarrollo está impulsada por una permanente intencionalidad: hacia el para qué de cualquier conducta consciente del sujeto.

Desde este punto de vista, el sujeto mediante la experiencia activa consigo mismo y con el entorno se va configurando su estilo de vida que se orienta hacia el fin o ideal. Estilo de vida que actuará como el dinamizador permanente en el proceso de socialización que imprimirá en el sujeto el sello característico de una determinada y estable forma de actuar; así es que dentro del estilo de vida encontramos que cada individuo es movido o impulsado por los fines que lo identifican, y que según Alfred Adler actúa inconscientemente y que el fin actúa como una búsqueda permanente de algo que en el plano conciente no existe, es conveniente hacer ésta precisión para distinguirlo de las metas y éxitos que involucran el plano consciente del sujeto.

Dicha distinción nos permite indicar que, ante los problemas que la vida misma plantea y según como el sujeto ha organizado su estilo de vida, es posible categorizar las formas como éste afronta y reacciona ante las diferentes circunstancias que a cada instante tiene que enfrentar.

Aprendizajes que se van constituyendo como elemento de control fundamental para el comportamiento en las actividades económicas, en lo que se refiere, al «estilo de vida». Es decir en las posturas fundamentadas al parecer de Adler con conceptos sinónimos: «personalidad», «yo», «sí mismo», «plan de vida», "actitud hacia la vida y hacia los otros», «lógica privada», «mundo privado», etc. o «conducta regulada» según Skinner, en Titze (1983, 126-128); así como la aplicación al ámbito organizacional hecha por Max Weber al 
atribuirle connotaciones sociales como «estilo social de vida», no por ello sin dejar de destacar que como lo advierte Adler que en el plano del «estilo personal» rige un tipo de lógica que difiere substancialmente de la lógica universal del common sense o de la razón (racionalidad).

Interpretándose como «estilo personal de vida» a la estructura global, donde los «complejos medios-fin» se constituyen en la manera de enfrentar el entorno o formalizar ante el contexto los proyectos de acción o enfrentamiento a la realidad como un «plan de vida» para el ajuste. Es así que Dreikurs hacia 1969,en Titze (1983, 123), señala que el plan de vida de una persona se identifica con su carácter «el plan de vida de un niño no se infiere de peculiaridades o de experiencias aisladas, sino del estilo que usa para superar las dificultades, reales o supuestas».

Por tanto, el «complejo medio fin» representa la unidad fundamental, desde la que se constituye los diversos proyectos del plan de vida; de ahí que cada plan esbozado encontrará los medios apropiados, asumiendo en la línea de acción del estilo personal aquello que le resulte efectivo.

Observándose dos categorías principales: El complejo medios-fin pasivo-regresivo y el complejo medios-fin activo-agresiva.

Para el caso de la primera categoría, los sujetos ubicados en ella emplean métodos pasivos y regresivos de seguridad existencial; presentando la «tendencia distanciadora» es decir trata de alcanzar el objetivo optando posturas de apartamiento de los problemas.

Éste apartamiento se efectúa en forma de distanciamiento espacial «tendencia a la retirada o a la fuga» (Künkel) o describiendóseles como distantes, hurañas, antipáticas, malhumoradas, intro-vertidas, arrogantes, desinteresadas, refractarias, etc. optan por una «actitud de erizo», instrumentándose para alejarse o inducir a que las personas que «le eviten».

Igualmente los medios-fin pasivo regresivo tiene la subforma de la «tendencia apelativa» es decir por lo general emplea el lenguaje no verbal como expresión de la propia debilidad y desamparo por ejemplo lágrimas, lamentaciones y quejas sobre la propia desgracia $\mathrm{y}$ de la ajena.

La categoría medio-fin activo-agresivo, los sujetos han adquirido la forma activa de buscar la seguridad existencial. Tienen como «presupuesto objetivo» el ejercicio de facultades o habilidades visomotoras, y como «presupuesto subjetivo» una auto imagen ya constituida (autovaloración o estima). Básicamente la actividad y la agresividad fortalece en el ajuste o enfrentamiento positivo y constructiva del medio. Observándose como tales «psicológicamente sanas».

Por tanto concluimos que el complejo medios-fin pueden ser productivos e improductivos, la primera tiene como fin el prójimo y la comunidad y la segunda tiene como fin a la persona en sí misma y un entorno cerrado que la distancia del prójimo y de la comunidad, a los que ve distantes, amenazadores o utilitariamente. De esta manera el estilo de vida se le puede categorizar en dos grupos, el primero denominado Orientación Productiva y el 
segundo Orientación Improductiva constituido por tres tipos que describiremos a continuación:

\section{A. ACTIVO AGRESIVO OBJETIVO}

Es el único Complejo Medios-fin propio del individuo productivo, tipo que se caracteriza por denotar el siguiente perfil:

- Actividad: Modifica la situación para que sea mejor.

- Agresividad: Enfrentamiento de los problemas cuando se presentan.

- Objetividad: Orientado a los objetivos, antes que, hacia las ganancias secundarias.

- Interés Social: Acciones que conducen a mejorar el medio en que vive.

- Autonomía: Capacidad de toma de decisiones por si mismo.

- Solución de Problemas: Mediante el diálogo y la comunicación.

- Relaciones Interpersonales: Genera interdependencias, así 1) Asume que tiene las capacidades necesarias y su imagen corporal le es satisfactoria.

2) Caracteriza a los demás, en especial a los que le rodean como básicamente bien intencionados y capaces, que merecen ser atendidos, respetados.

- Se expresa a través de la fórmula: «Yo estoy bien-Tú estás bien», es decir Autoimagen positiva-Heteroimagen positiva.

\section{B. ACTIVO AGRESIVO DFSTRUCTIVO}

Es el Complejo Medio Medios-fin improductivo con menor deterioro por parte del sujeto o con mejor pronóstico. Tipo que se caracteriza por denotar el siguiente perfil:

- Actividad: Intenta cambiar las cosas hacia la solución.

- Agresividad: Enfrentamiento de los problemas cuando se presentan.

- Destructividad: Uso de medios más bien violentos, en los que intenta hacer resaltar su participación y su imagen, afanes exhibicionistas.

- Interés Social: Moderadamente deteriorado; sus acciones cuidan más la propia imagen antes que mejorar el medio en que vive.

- Solución de Problemas: Lo realiza mediante la fuerza y la imposición.

- Autonomía: Es restringida, se deja llevar por sus necesidades emocionales.

- Relaciones Interpersonales:

1) Asume que tiene las capacidades necesarias y su imagen corporal le es satisfactoria.

2) Caracteriza a los que le rodean como básicamente mal intencionados e incapaces, culpables de lo malo en el mundo de quienes es mejor desconfiar.

- Se expresa a través de la fórmula: «Yo Estoy Bien-Tú Estás Mal», es decir Autoimagen Positiva-Heteroimagen Negativa respectivamente. 


\section{PASIVO REGRESIVO APELATIVO}

Es el Complejo Medios-Fin improductivo con deterioro medio por parte del sujeto y con pronóstico reservado. Tipo que se caracteriza por denotar el siguiente perfil:

- Pasividad: No se esfuerza por cambiar las cosas, aún que le perjudiquen.

- Regresividad: Retroceso frente a los problemas y elaboración de síntomas como excusa por su evasión.

- Apelatividad: Espera de los otros sea por compasión paren tal o moral le resuelvan sus problemas.

- Interés Social: Gravemente deteriorado; sus acciones tratan de asegurar la atención de quienes le rodean sin importarle el costo individual y grupal de las acciones efectuadas.

- Solución de Problemas: Lo realiza con ayuda de otros.

- Autonomía: Esta es muy restringida, su necesidad de ayuda le convierte en dependiente en la toma de decisiones.

- Relaciones Interpersonales:

1) Asume que no tiene las capacidades necesarias, su imagen corporal le es insatisfactoria, no ejerce la autoridad y los derechos que los diferentes roles le otorgan.

2) Caracteriza a los que le rodean como básicamente poderosos con la posibilidad de ayudarlo, y orientados a su favor.

- Se expresa a través de la formula: "Yo Estoy Mal-Tú Estás Bien», es decir Autoimagen Negativa-Heteroimagen Positiva, respectivamente.

\section{PASIVO REGRESIVO DISTANCIADOR}

Es el Complejo Medios-Fin improductivo con deterioro elevado por parte del sujeto, propio de las neurosis graves y de las psicosis. Su pronóstico es definitivamente malo, debido al profundo retraimiento que se observa en el individuo. Tipo que se caracteriza por denotar el siguiente perfil:

- Pasividad: No se esfuerza por cambiar las cosas aún cuando lo perjudiquen.

- Regresividad: Retroceso frente a los problemas y elabora síntomas -excusa- por su evasión.

- Distanciamiento: Alejamiento psicológico o físico de la realidad, ya que no espera nada de los otros. Desarrolla estrategias para alejar a los demás a la manera de puercoespín.

- Interés Social: Profundamente deteriorado, sus acciones lo alejan de quienes lo rodean interés en su mundo de aislamiento-o En sus acciones no hay el mínimo intento de mejorar el entorno, y parece poco interesado en que el entorno lo mejore.

- Solución de Problemas: Renuncia total.

- Autonomía: Carencia total, sujeto sin acción autodirigida sobre el mundo.

- Relaciones Interpersonales:

1) Asume que no tiene las capacidades necesarias, su imagen corporal le es insatisfactoria, no ejerce la autoridad y los derechos que los diferentes roles le dan -debilidad de carácter-o 2) Caracteriza a los que le rodean como sujetos incapaces o malvados, donde la imagen de buenos sólo es un disfraz.

- Se expresa a través de la formula: «Yo Estoy Mal-Tú Estás Mal», es decir Autoimagen Negativa-Heteroimagen Negativa, respectivamente. 
Veamos ahora el segundo componente de nuestro investigación, la teoría atribucional la misma que a partir de los estudios y propuestas teóricas de Heider (1956), el estudio de las estrategias de afronte ante situaciones adversas, han considerado las explicaciones que los propios agentes realizan de las razones por las cuales tienen éxito o no al procurarse una actividad en el mercado laboral.

La atribución para categorizar la situación del trabajo de los miembros de la comunidad está relacionada al estilo de vida, formada por el aprendizaje, el que otorga o posibilita respuestas de logro o fracaso, de acuerdo al estilo de explicarse las causas de los éxitos y fracasos.

Cada miembro de la comunidad según los diferentes grupos de pertenencia o referencia, le da significado al empleo, subempleo o desempleado. De tal forma cada uno de éstas categorías de situación laboral cobra motivación según la percepción moldeada por la atribución, denotando connotaciones propias en dichos individuos.

Según Peterson \& Stunkard (1992), el estilo atribucional, es un constructo mas comprehensivo y con mayor poder explicativo, que el de Locus de Control formulado por J. B. Rotter, y el de Autoeficacia de A. Bandura. Aunque no los sustituya totalmente, comparte con estas y otras teorías el explicar los procesos cognitivos del control personal que se reflejan en la conducta motivada para el empleo.

El control personal es uno de los modos importantes en que las personas se diferencian unas de otras. Reside en la transacción entre la persona y el mundo, que en este caso se refiere a su situación laboral. No es sólo una disposición $\mathrm{O}$ sólo una característica del ambiente puesto que toma la forma de las creencias de que uno puede afectar los resultados reales, afrontar consecuencias. El caso más estudiado y documentado se encuentra en los estudios de Seligman (1978) sobre desesperanza aprendida. Seligman, Ardila y otros estudiaron el fenómeno de la falta de iniciativa para enfrentar las situaciones adversas o de alto nivel de tensión. Según sus resultados, las personas, dependiendo de ciertas características de su personalidad, generarán en mayor o menor medida el cuadro de desesperanza.

Asimismo, las investigaciones de Mc Clelland (1968) nos informan que los contextos con bajos niveles de libertad individual generan en los niños, personalidades inmaduras que no adquieren ni constituyen en su personalidad un grado alto de la motivación de logro, la misma que caracteriza la realización o producción de los agentes del desarrollo económico sostenido (Paredes, 1997) es decir la que es propia de las personalidades con estilo activo agresivo objetivo.

Abordaremos el tema desde la perspectiva de la teoría de la atribución porque tiene importantes contribuciones para entender la motivación de logro y su influencia en el desempeño y el incremento del rendimiento en las actividades laborales y de diversa Índole.

Desde la teoría reformulada de la desesperanza aprendida (Seligman, 1975). Se han originado los programas de investigación responsables de la abundante producción sobre estilo 
atribucional e incremento del desempeño (Peterson, 1992).

El estilo explicativo llega a ser de tal nivel de generalidad que incluso ha sido postulado como un rasgo constitutivo de fa personalidad (Reeve, 1995).

Además el estilo explicativo pesimista está asociado a la depresión e incluso a la presencia persistente de enfermedades (Peterson,1992). Los resultados de un estilo explicativo pesimista según diversas investigaciones abarcan: la depresión (Sweeney, Anderson, \& Bailey, 1986), bajas calificaciones en la escuela (Peterson \& Barret, 1987), soledad (Anderson, Horowitz, \& French, 1983), mal estado de salud física (Peterson, Seligman \& Vaillant, 1988), y lo más importante de cara al presente estudio también se han encontrado relaciones entre el bajo desempeño laboral y el estilo atribucional (Seligman \& Schulman, 1986).

Al relacionar las atribuciones con la motivación y el desempeño en situaciones competitivas, se ha encontrado que están relacionadas con los estilos atribucionales. Anderson (1980) afirma que las primeras investigaciones fueron en situaciones con tareas típicas de laboratorio, sin embargo han sido más bien en las situaciones interpersonales más parecidas a la vida diaria que se ha encontrado mayor relación. Esto es entendible porque la complejidad y variedad de causas de tales situaciones hace que los procesos cognitivos de atribución jueguen un rol más importante que en las tareas sencillas de laboratorio.

Bernard Weiner (1992) postuló que las personas suelen atribuir sus éxitos o fracasos a una de las siguientes cuatro posibles causas: habilidad, cantidad de esfuerzo, dificultad de la tarea y la suerte. Ellas se integran en dos dimensiones: Estable-Inestable, e Interno-Externo. La habilidad es entonces una causa interna al sujeto y relativamente estable en el tiempo, la cantidad de esfuerzo es el otro elemento de la dimensión interna pero que puede fácilmente variar de una situación a otra, es decir no es estable en el tiempo. Mientras que la dificultad de la tarea es un aspecto externo al sujeto y relativamente estable mientras que el factor suerte es externo y no constante o inestable pues no está bajo control de la persona y puede variar de una ocasión a otra. Weiner presenta variados resultados de investigaciones que muestran la influencia de las atribuciones en la persistencia, intensidad y la elección de determinadas conductas.

Peterson (1991) incluyen además de las dos dimensiones de atribución consideradas por Weiner, una tercera que considera lo global o especifico del factor causa!. El estilo atribucional es una variable cognitiva de personalidad que refleja el modo en que las personas explican habitualmente las causas de sus fracasos, da cuenta de la falta de motivación o desmoralización ante situaciones que demandan logros (Peterson, 1990). Este efecto sería por ello tan general que minaría las percepciones de la estima personal.

Una explicación interna, estable y global, ante un resultado adverso o negativo, es la que imprime mayor pesimismo y desmoralización, en el individuo y es denominada por ello estilo explicativo pesimista. En el otro extremo está el estilo optimista que sería el que mejor predispone a la persona para persistir en sus intentos de conseguir una ocupación y de mejorar su situación laboral en general.

Por lo descrito hasta ahora es importante en las condiciones presentes analizar la 
situación de situación del empleo y desempleo.

La permanente aceleración del fenómeno de la mundialización y del progreso técnico suscita a la vez problemas y oportunidades, precisamente cuando la situación del empleo no deja de ser sombría y en la mayoría de los países persiste un fuerte desempleo y subempleo. Según el informe de la Oficina Internacional del Trabajo (OIT) en los dos últimos años el empleo no ha mejorado, aproximadamente 1,000 millones de trabajadores (un tercio de la población activa del mundo) están desempleados o subempleados, en el caso peruano según el informe sobre el empleo en el mundo 1998-99 de la OIT la tasa de desempleo llega al 9.5.

Situación laboral sumada a las crisis económicas como las denominadas «Vodka» $\mathrm{y}$ «Asiática», y a los efectos del Fenómeno del Niño, ocurridos en el presente año, tienen repercusiones, aún más severas al contraerse la inversión; por ende disminuye la oferta del empleo formal y decae la calidad del empleo o el buen empleo, para dar lugar a nuevas formas de empleo que constituyen el sector no estructurado caracterizado por la inseguridad y bajos salarios, a pesar de los esfuerzos que realiza la pequeña y mediana empresa como generadores del buen empleo (productivo) en nuestra realidad.

Al igual que la OIT otras organizaciones internacionales tales como OLDEPESCA, CEPAL, subrayan la creciente evidencia de que la educación y los niveles de destreza de la mano de obra son factores determinantes de éxito o fracaso, instando a una estrategia «activista» de los recursos humanos para hacer frente a los retos de la competitividad mundial, donde la escuela y los centros de formación profesional tienen la gran responsabilidad de formar cada vez más personas, con la humanidad de ser capaces de lograr la mejor relación costo-eficacia para enfrentar los complejos sistemas de producción y tecnologías, por otra parte crear una mano de obra adaptable, facilitada por ambientes psicosociales que propicien personalidades maduras, para solventar las necesidades de un mercado en rápido cambio.

La formación de las personas como se observa depende de los actores interactuantes, es decir, de las características no sólo técnicas del profesor, ni sólo de las habilidades del estudiante, sino de otros factores complementarios, entre ellos, el psicológico. Procesos psicológicos mediadores tal vez decisivos, tal como la personalidad y las motivaciones.

Al respecto en la investigación del autor y colaboradores (IIPsi, 1991) en estudiantes universitarios se encontró que el tipo personalidad predominante es hacia la orientación productiva, de acuerdo al Complejos Medio-Fin del tipo Activo Objetivo Agresivo. Siguiendo (IIPsi, 1992) en la misma línea de trabajo, centrado el interés, hacia las disposiciones psicológicas del joven en la perspectiva del desarrollo del país, obteniéndose como resultado que, los sujetos con orientación productiva presentan un mayor apego institucional y compromiso de desarrollo con la misma. Nuestra investigación (IIPsi, 1997) sobre la motivación de logro según Mc Clelland y las disposiciones psicológicas para asumir el rol empresarial, tiene por resultado que el $90 \%$ de los estudiantes sanmarquinos presentan disposiciones favorables pero asociadas a una motivación de filiación, así mismo Paredes (1997) encontró que los empresarios del sector pesquero peruano su motivación de logro es promedio, nivel que no facilita un desarrollo económico sostenido. En la investigación del autor y colaboradores (IIPsi., 1998) se describe con respecto a la población de Lima Metropolitana, que las variables poblacionales, entre ellas la Ocupación, no influyen sobre las disposiciones psicológicas hacia los estados de emergencia de origen natural y social; observándose miedo y 
con reacciones de lograr la protección del hogar para luego solicitar ayuda .

Bajo estas circunstancias involucradas en estado de crisis, fundamentalmente económica, las personas excluidas o no del colectivo social, reaccionan de alguna forma, entendiéndose que tales reacciones no son meramente circunstanciales sino que obedecen a toda una configuración, donde se combinan las habilidades, actitudes y valores que hace observable su resultado a través del comportamiento individual (personalidad) y que llamamos afronte.

De esta manera queremos despejar la siguiente interrogante: ¿Se relaciona los Complejos Medios-Fin con los tipos de afronte ante la Crisis Económica? en una muestra de limeños comprendidos entre los 15 a 45 años de edad en diferentes condiciones estructuradas o no de empleo.

La presente investigación permitirá conocer en jóvenes y en adultos la forma como los Complejos Medios-Fin se relacionan con los tipos de afronte ante la crisis económica desde la teoría atribucional.

Se demostrará las intensidades y direccionalidad de las relaciones entre los Complejos Medios-Fin: Orientación Productiva, frente a los tipos de Orientación Improductiva, con los tipos de afronte hacia la crisis económica en el contexto del empleo.

De los hallazgos se estará en condiciones de plantear recomendaciones sobre todo para los agentes de capacitación y gestión para que asuman medidas para el manejo apropiado de las variables motivacionales acorde con los Complejos Medios-Fin.

\section{HIPÓTESIS}

H1 La orientación productiva de los complejos medio-fin se relaciona inversa negativamente con el tipo de afronte ante la crisis económica de estilo atribucional pesimista.

H2 La correlaciones entre los complejos medios-fin con el tipo de afronte ante la crisis económica difieren significativamente según la condición de empleado o desempleado.

H3 La correlaciones entre los complejos medio- fin con el tipo de afronte ante la crisis económica difieren significativamente según el género.

\section{METODOLOGIA}

Población y Muestra, la población referencial es la de Lima Metropolitana, la misma que según el censo de 1993 (INEI 1993) esta constituido por 3987003 de los cuales 1927248 son varones y 2059755 son mujeres comprendidos entre los rangos de 15 a más de 45 años de edad.

El tamaño de la muestra fue calculada a partir del total poblacional de Lima para los rangos de 15 hasta 45 a más años de edad y por conglomerados en función al género, cuya cifra es de tres millones novecientos ochenta y siete mil tres ciudadanos ( 3987 003), del cual se estimó un tamaño de muestra con un error no mayor del 0.05 y un nivel de confianza del 0.95 , constituido por 483 sujetos, cuya distribución fue la siguiente: 
Tabla No. 1

Distribución porcentual de la muestra en función al género y estructura laboral y rango de edad de Lima

\begin{tabular}{|c|c|c|c|c|c|c|c|c|}
\hline \multirow{2}{*}{$\begin{array}{c}\text { Rango de } \\
\text { edad }\end{array}$} & \multicolumn{4}{|c|}{ VARONES } & \multicolumn{4}{|c|}{ MUJERES } \\
\hline & \multicolumn{2}{|c|}{ Empleados } & \multicolumn{2}{|c|}{ Desempleados } & \multicolumn{2}{|c|}{ Empleados } & \multicolumn{2}{|c|}{ Desempleados } \\
\hline $15-19$ & 27 & 5.59 & 11 & 2.28 & 27 & 5.59 & 14 & 2.9 \\
\hline $20-24$ & 19 & 3.93 & 18 & 3.73 & 26 & 5.38 & 14 & 2.9 \\
\hline $25-29$ & 20 & 4.14 & 12 & 2.48 & 20 & 4.14 & 14 & 2.9 \\
\hline $30-34$ & 17 & 3.52 & 10 & 2.07 & 15 & 3.10 & 13 & 2.69 \\
\hline $35-39$ & 12 & 2.48 & 10 & 2.07 & 12 & 2.48 & 13 & 2.69 \\
\hline $40-44$ & 11 & 2.28 & 8 & 1.65 & 11 & 2.28 & 9 & 1.86 \\
\hline 45 a más & 47 & 9.73 & 11 & 2.28 & 49 & 10.14 & 132 & 27.33 \\
\hline Sub-parcial & 153 & 31.68 & 80 & 16.56 & 160 & 33.13 & 90 & 18.63 \\
\hline Sub-Total & \multicolumn{2}{|c|}{233} & \multicolumn{2}{|c|}{$48.24 \%$} & \multicolumn{2}{|c|}{250} & \multicolumn{2}{|c|}{$51.79 \%$} \\
\hline TOTAL & \multicolumn{8}{|c|}{483} \\
\hline
\end{tabular}

La recolección de información fue de dos formas, la primera colectiva en grupos no mayores de 25 por vez, para centros laborales que tuvieron la gentileza de concedemos 45 minutos para la aplicación de ambas pruebas.

La segunda forma y la que más se utilizó fue la autogenerada, para la cual se contactaba con una persona ubicada dentro de los rangos de edad y que esté en condición laboral de empleado o desempleado y luego se le solicitaba que nos hiciera contacto con un conocido, en estos casos la aplicación fue individual. Es importante destacar que fue esporádico encontrar sujetos en condición de subempleo y a partir de ellos no fue posible autogenerar muestra, razón por la cual no fue considerado esta categoría en la presente investigación, como hubiéramos querido.

\section{MÉTODOS Y MATERIALES}

La presente investigación es descriptiva correlacional en cuanto se pretende conocer la intensidad y direccionalidad de la relaciones entre los Complejos Medios-Fin del estilo de vida desarrollada por Alfred Adler con los tipos de afronte ante el estado de crisis económica desde la perspectiva del estilo atribucional.

La recolección de datos fue utilizando el examen psicométrico para lo cual se aplicaron a la muestra dos pruebas tipo cuestionario, el primero de ellos es el Inventario Monofactorial de Complejos Medios-Fin elaborada en 1991 por el autor y colaboradores, instrumento que cuenta con validez de contenido por criterio de jueces y Confiabilidad mayor de 0.90 por el método de la consistencia interna y de 0.88 por el método de test retests.

La segunda prueba utilizada para medir el afronte ante el estado de crisis económica desde la perspectiva del estilo atribucional se ha utilizado el Cuestionario de Estilo Atribucional (ASQ: Attributional Style Questionnaire). El cuestionario evalúa el estilo 
explicativo mediante el reporte del propio sujeto. En la versión expandida del cuestionario (Peterson, \& Villanova, 1988). Se le presentan veinticuatro situaciones hipotéticas de fracaso. En cada una de ellas debe indicar con sus propias palabras cual cree que es la causa principal de que las cosas hayan resultado mal y entonces debe puntuar esa causa en cada una de las tres dimensiones del estilo explicativa: internalidad, estabilidad, globalidad y el complejo negativo, en una escala del uno al siete.

Para el presente estudio hemos calculado la confiabilidad de la escala por dos métodos el de la consistencia interna y por el método del test retest con un grupo piloto de 280 sujetos de los cuales 80 tenían entre 15 a 25 años de edad, 120 estaban entre los 26 a 35 años y 8 sujetos entre los 36 a los 45 años de edad, a su vez 120 fueron desempleados y 160 empleados. Los coeficientes se calcularon para cada una de las dimensiones encontrando los siguientes resultados:

\begin{tabular}{|l|c|c|}
\hline Dimensiones & $\begin{array}{c}\text { Consistencia } \\
\text { Interna }\end{array}$ & $\begin{array}{c}\text { Test } \\
\text { Retest }\end{array}$ \\
\hline Interno-Externo & 0.88 & 0.90 \\
Estable-Inestable & 0.89 & 0.89 \\
Global-Específico & 0.88 & 0.87 \\
\hline
\end{tabular}

La validez de la escala para el presente estudio fue mediante el análisis de contenido efectuado de dos maneras, el primero con la consulta de expertos, mediante el cual se retuvieron los ítemes que al 0.05 de significación mediante la prueba del Ji cuadrado los Jueces aprobaran los encabezados de cada uno de los doce ítemes de cada una de las dimensiones.

\section{RESULTADOS}

Como vimos los Complejos Medios-Fin del estilo de vida que según A. Adler define la personalidad del sujeto pueden ser productivas e improductivas esta segunda diferencia a los sujetos distanciadores, apelativos y destructivos. Por su parte los estilos atribucionales identifican la causa que el sujeto concede a una situación específica, en nuestro estudio fue ante indicadores de crisis económica, las que pueden ser de carácter externo, cuando el individuo atribuye que la causa se debe a otro o a cualquier otra circunstancia menos a él mismo, que vendría a ser la causa interna.

Por otro lado el sujeto puede atribuir que tal causa no se volverá a presentar, en este caso hay inestabilidad, frente a la estabilidad donde por lo contrario considera que siempre estará presente.

La tercera dimensión atribucional es la especificidad, cuando se sume que tal causa solo influye en una situación concreta, su opuesto de esta dimensión es la globalidad, aquí el sujeto asume que ésa causa influye en todas las situaciones de la vida.

Combinando las tres dimensiones atribucionales: Externo-Interno, Inestable-Estable, Específico y Global, tenemos como resultado el estilo atribucional que define al sujeto con 
estilo atribucional Pesimista u Optimista.

Una explicación interna, estable y global, ante un resultado adverso o negativo, es la que imprime mayor pesimismo y desmoralización, en el individuo y es denominada por ello estilo explicativo pesimista. En el otro extremo está el estilo explicativo optimista que sería el que mejor predispone a la persona para persistir en sus intentos de conseguir una ocupación y de mejorar su situación laboral en general.

Para despejar la interrogante planteada en torno a la existencia o no de correlación directa positiva entre los complejos medio-fin con el estilo atribucional y si tanto la condición de empleado o desempleado como el género plantean diferencias en las correlaciones entre la personalidad y los estilos atribucionales hemos sometido los datos de los cuestionarios aplicados a la muestra al análisis de regresión de Pearson, luego a la contrastación de correlaciones. Complementariamente hemos contrastado, mediante la razón crítica de diferencia de medias los resultados obtenidos en cada una de las dimensiones y en el compuesto negativo del estilo atribucional y de los complejos medios fin, y finalmente hemos creído conveniente describir a la muestra estudiada porcentual mente en función al tipo de personalidad y del compuesto negativo del estilo atribucional, resultados que a continuación presentamos.

a) Los Complejos medios-fin y su relación con los estilos atribucionales, para este análisis hemos correlacionado cada una de las dimensiones del estilo atribucional con cada tipo de personalidad encontrando lo siguiente:

a.I La dimensión del estilo atribucional Externo - Interno presenta correlaciones significativas con el Tipo Apelativo (- 0.54) Y con el tipo Destructivo (0.36), esto significa que los sujetos que se caracterizan por hacer que otros hagan lo que el debería hacer presentan un mayor afronte atribucional Externo. Los tipo destructivos se caracterizan por presentar un mayor afronte del tipo Interno. Por su parte el Tipo productivo presenta una correlación de 0.15 que siendo baja es significativa al 0.05 de error, lo que significa que débilmente se relacionas la dimensión interna con el tipo que se caracteriza por asumir sus responsabilidades dentro de un sentido de comunidad.

a.2 La dimensión Inestabilidad estabilidad se correlaciona con los tipos Distanciadores, apelativos y Productivos con los coeficientes de correlación: 0.61, 0.96 y 0.78 respectivamente, todas ellas muy significativas aún para el 0.01 de error, lo que significa que estos tres tipos de personalidad afrontan a los estados de crisis considerando estables las causas supuestas por ellos mismos.

a.3 La dimensión Específico-Global correlaciona significativamente con los tipos: Distanciadores (0.95), con el tipo apelativo (0.78) y con el tipo productivo (0.69) lo que significa que existe tendencia para considera que la supuesta causa de la crisis influye en todas las situaciones de sus vidas. Leyendo las intensidades de las correlaciones notamos que es muy alto en los Distanciadores y Apelativos y con menor intensidad en los productivos.

a.4 En el compuesto negativo encontramos un coeficiente de correlaciones de 0.90 con el 
tipo distanciador, 0.95 con el tipo Apelativo, 0.11 con el tipo Destructivo y de -0.35 con el tipo Productivo. Se nota que los dos primeros tipos tiene un estilo atribucional pesimista, con el tipo Destructivo no hay correlación significativa por ende los niveles de pesimismo y optimismo son dispersos en este tipo. En el tipo Productivo la dirección negativa indica presencia de relación inversa por tanto a mayor productividad menos pesimismo y a mayor pesimismo menor productividad, resultado que puede verse en la Tabla Nro. 2.

Tabla $\mathbf{N}^{0} 2$

de correlaciones entre los tipos personalidad con los estilos atribucionales de afronte ante la crisis obtenida con una muestra de 483 sujetos entre los 19 a mas de 45 años de edad de Lima Metropolitana

\begin{tabular}{|l|c|c|c|c|}
\hline \multirow{2}{*}{ Est. Atribución } & \multicolumn{4}{|c|}{ Personalidad } \\
\cline { 2 - 5 } & Distanciador & Apelativo & Destructivo & Productivo \\
\hline Externo-Interno & -0.02 & $-0,54^{*}$ & $0,36^{*}$ & $0,15^{*}$ \\
Inestable-Estable & $0,61^{*}$ & $0,96^{*}$ & 0.09 & $0,78^{*}$ \\
Específico & $0,95^{*}$ & 0.78 & 0.07 & $0,69^{*}$ \\
Importancia & $0,62^{*}$ & 0.1 & 0.06 & 0.18 \\
Compuesto negativo & $0,90^{*}$ & $0,95^{*}$ & 0.11 & $-0,35^{*}$ \\
\hline
\end{tabular}

(*) Correlación significativa al 0.05 de margen de error

b) Contrastación de las correlaciones entre los complejos medio-fin con el estilo atribucional en sujetos en condición de empleo y en condición de desempleo, para este propósito se calculó la correlación de Pearson para cada tipo de personalidad con la puntuación del compuesto negativo que hacer referencia al estilo. atribucional pesimista. Estos coeficientes se calcularon por separado en función a la condición de empleado y desempleado, luego con el objeto de averiguar si las correlaciones difieren en base a ésa variable, convertimos los coeficientes a puntuaciones zeta (z) para calcular la razón crítica de correlaciones (RC) para un nivel de confianza del $95 \%$.

Efectuado el análisis encontramos:

b.l Los empleados con mayores indicadores de personalidad productiva tienen menor puntuación en el compuesto negativo o tienen menores atribuciones pesimistas respecto a los desempleados, lo que deja entrever que en este grupo hay una tendencia al pesimismo en relación a la crisis económica ya que aún cuando la correlación es solo de 0.18 tiene un valor t igual a 2.37 que resulta significativa al 0.05 de error. En suma los empleados enfrentan a la crisis económica con optimismo en tanto que los desempleados tienden al pesimismo.

b.2 Los empleados con personalidad apelativa no difieren de los desempleados con personalidad similar, lo que significa que el estilo atribucional pesimista se presenta de manera muy similar en ambos grupos.

b.3 Los desempleado s con mayor indicadores de personalidad distanciadora tienen mayor puntuación en el compuesto negativo o tienen mayores atribuciones pesimistas que los 
distanciadores en condición de empleo. Es importante señalar que ambos grupos los coeficientes hallados son altos y directos positivos dejando entrever que el tipo de personalidad distanciadora se relaciona con el estilo atribucional pesimista y que esta se acentúa en condiciones de desempleo.

b.4 La correlación hallada en los empleados con una personalidad destructiva es baja pero significativa al $95 \%$ de confianza de manera similar que del grupo de desempleados, aclarando que en éstos el coeficiente es inverso negativo (-0.18), la razón crítica calculada arroja un valor de -2.85 que resulta muy significativa al 0.05 de margen de error, esto significa que en los desempleados se espera encontrar que a mayor indicadores de personalidad destructiva habrá más indicadores de afronte de estilo pesimista, hecho que no se encuentra en el grupo de empleados.

La interpretación de estos resultados (Tabla No. 3) es la siguiente: Los empleados tienden a enfrentar la crisis con estilo optimista al contrario del grupo de' desempleados, de lo que se infiere que de alguna manera la combinación de personalidad y estilo atribucional tiene que ver con estar empleado o desempleado, lo que llevaría a reflexionar que siendo la personalidad muy resistente al cambio en comparación con el estilo atribucional, una manera de contribuir con la solución del problema sería desarrollando y comprobando la eficiencia de programas que apuntes a modificar los estilos atribucionales

\section{Tabla Nro 03}

De diferencia de correlaciones entre los tipos de personalidad con el estilo atribucional pesimista en función a la condición de empleo (313 sujetos) y desempleo (170 sujetos).

\begin{tabular}{|l|c|c|c|r|r|r|r|}
\cline { 2 - 7 } \multicolumn{1}{c|}{} & \multicolumn{2}{c|}{ Empleado } & \multicolumn{2}{c|}{ Desempleado } & \multirow{2}{*}{ Dif } & GD & \multirow{2}{*}{ RC } \\
\cline { 2 - 8 } & $\mathbf{~ r ~}$ & $\mathbf{z}$ & $\mathbf{r}$ & $\mathbf{z}$ & & \\
\hline $\begin{array}{l}\text { Productivo con Estilo } \\
\text { atribucional Pesimista }\end{array}$ & -0.35 & -.036 & 0.18 & 0.18 & -.054 & 0.13 & $-4.15^{*}$ \\
$\begin{array}{l}\text { Apelativo con Estilo } \\
\text { atribucional Pesimista }\end{array}$ & 0.92 & 1.59 & 0.95 & 1.83 & 0.24 & 0.13 & 1.85 \\
$\begin{array}{l}\text { Distanciador con Estile } \\
\text { atribucional Pesimista }\end{array}$ & 0.80 & 1.10 & 0.90 & 1.47 & 0.37 & 0.13 & $2.85^{*}$ \\
$\begin{array}{l}\text { Destructivo con Estilo } \\
\text { atribucional Pesimista }\end{array}$ & 0.19 & 0.19 & -0.18 & -0.18 & -0.37 & 0.13 & $-2.85^{*}$ \\
\hline
\end{tabular}

(*) razón crítica significativa al 0.05 de error.

c) Contrastación de las correlaciones entre los complejos medios-fin con el estilo atribucional en función al género, en el análisis anterior hemos visto la forma como las variables personalidad y estilos atribucionales se relacionan en función a la condición de empleado y desempleado, ahora veremos la forma como estas variables se relacionan en función al género. Hacemos este análisis para averiguar si en función al género las relaciones personalidad y estilo atribucional varían significativamente. Para este fín hemos calculado 
la razón crítica de la diferencia de correlaciones, encontrando como resultado que en ningún caso se ha registrado diferencias significativas de las correlaciones entre las variables personalidad y estilos atribucionales.

c.1 De lo hallado se concluye que las relaciones entre los tipos de personalidad y los estilos atribucionales son similares entre varones y mujeres, en ambos se espera que cuanto tengan mayores indicado-res de una personalidad productiva habrá menor riesgo de desesperanza o pesimismo ante la crisis económica.

c.2 Los tipos apelativos y distanciadores, presentan mayor riesgo para desarrollar estilos pesimistas ante los estados de crisis económica.

c.3 Los tipos destructivos dejan entrever que a mayor indicadores de personalidad destructiva habrá menor número de indicadores de compuesto negativo o estilo pesimista, este resultado es algo similar a lo hallado con la personalidad del tipo productiva, esta semejanza se debe a que ambos tienen en común la actividad para enfrentar las cosas de la vida, ambos son de iniciativa y de embarcarse a la solución de los problemas, la diferencia radica en que mientras los productivos actúan resolviendo problemas y al mismo tiempo desarrollando su autorrealización y promoviendo el desarrollo de los otros y la comunidad, en tanto que los sujetos de orientación productiva experimentan el aquí y el ahora, son inmediatistas y pueden fácilmente pasar por alto los derechos de los demás, es que lo mas importante es resolver el problema ahora.

Los resultados se ven en la Tabla No. 04

Tabla No. 04

De diferencia de correlaciones entre . los tipos de personalidad con el estilo atribucional pesimista en función al género (233 varones) y (250 mujeres).

\begin{tabular}{|c|c|c|c|c|c|c|c|}
\hline & \multicolumn{2}{|c|}{ Mujeres } & \multicolumn{2}{|c|}{ Varones } & \multirow{2}{*}{ Dif } & \multirow{2}{*}{$G D$} & \multirow{2}{*}{$R C$} \\
\hline & $r$ & $z$ & $r$ & $z$ & & & \\
\hline $\begin{array}{l}\text { Productivo con Estilo } \\
\text { atribucional Pesimista }\end{array}$ & -0.33 & 0.34 & -0.35 & 0.36 & -0.02 & 0.09 & -0.22 \\
\hline $\begin{array}{l}\text { Apelativo con Estilo } \\
\text { atribucional Pesimista }\end{array}$ & 0.90 & 1.47 & 0.92 & 1.59 & 0.12 & 0.09 & 1.33 \\
\hline $\begin{array}{l}\text { Distanciador con Estilo } \\
\text { atribucional Pesimista }\end{array}$ & 0.84 & 1.12 & 0.80 & 1.10 & 0.04 & 0.09 & 0.44 \\
\hline $\begin{array}{l}\text { Destructivo con Estilo } \\
\text { atribucional Pesimista }\end{array}$ & -0.15 & 0.15 & -0.18 & 0.80 & -0.03 & 0.09 & $-0.33+$ \\
\hline
\end{tabular}


d) Contrastación de las medias aritméticas de los tipos de personalidad y estilos atribucionales en función a la condición de empleado y desempleado, para este análisis hemos evaluado y diagnosticado a cada sujeto en función al tipo de personalidad y estilo atribucional, luego dentro de cada grupo hemos obtenido la media aritmética y desviación estándar con el propósito de ubicarlos dentro del continum de indicadores el grado en que se ubica tanto los empleados como los desempleados, de modo que nos permita efectuar las comparaciones con la intención de verificar si en función a la condición de empleo o desempleo difieren los estilos atribucionales y los tipos de personalidad de modo que nos permita inferir que en alguna medida, la personalidad y los estilos atribucionales inciden dirigiendo al sujeto para enfrentar los problemas de forma eficiente y eficaz.

Al efectuar el análisis comparativo de la medias aritméticas para los diferentes tipos de personalidad y estilos atribucionales en función a la condición de empleo y desempleo, encontramos:

d.1 Los sujetos apelativos, distanciadores y destructivos en condición de empleo no difieren significativamente de los sujetos con tipo similar y en condición de desempleado, aun cuando se observa que la media aritmética del tipo destructivo tiende a ser mayor en el grupo de empleados, lo que es entendible por su fuerte componente de actividad. En tanto que la media de los apelativos es mayor en los desempleados.

d.2 La media aritmética del tipo productivo es significativamente mayor en los empleados que en los sujetos en condición de desempleo.

d.3 Al contrastar la media aritmética de compuesto negativo que define el estilo atribucional pesimista encontramos que los des empleados se caracterizan por explicar los acontecimientos de forma pesimista respecto a los sujetos en condición de empleo que se muestran optimistas. Este resultado nos dice, además, que los desempleados se caracterizan por culparse a si mismos, para mantener las causas de su estado actual como estables y que compromete a toda su vida tal como puede verse en la Tabla No.5 
Tabla No 5

Diferencia de medias aritméticas de las puntuaciones obtenidas para cada tipo de personalidad y estilo atribucional en función a la condición de Empleo (313) y desempleo (170)

\begin{tabular}{|c|c|c|c|c|c|c|c|c|c|}
\hline \multirow{3}{*}{$\begin{array}{c}\text { Personalidad y } \\
\text { Atribución }\end{array}$} & \multicolumn{9}{|c|}{ Condición Laboral } \\
\hline & \multicolumn{3}{|c|}{ Empleados } & \multicolumn{3}{|c|}{ Desempleados } & \multirow{2}{*}{ Dif } & \multirow{2}{*}{ GD } & \multirow{2}{*}{$\mathbf{R C}$} \\
\hline & $\mathbf{N}$ & $\mathbf{X}$ & DS & $\mathbf{N}$ & $\mathbf{X}$ & DS & & & \\
\hline Distanciador & 19 & 3.1 & 1.45 & 10 & 4.38 & 2.25 & 0.68 & 0.79 & 0.86 \\
\hline Apelativo & 56 & 3.32 & 1.45 & 4.13 & 4.13 & 2.08 & 0.68 & 0.79 & 0.86 \\
\hline Destructivo & 10 & 5.43 & 2.28 & 3.78 & 3.78 & 1.95 & 0.68 & 0.79 & 0.86 \\
\hline Productivo & 228 & 4.7 & 1.42 & 3.25 & 3.25 & 1.89 & 0.68 & 0.79 & 0.86 \\
\hline Compuesto Negativo & 72 & 3.18 & 1.4 & 3.95 & 3.95 & 1.45 & 0.68 & 0.79 & 0.86 \\
\hline
\end{tabular}

e) Descripción porcentual de los tipos de personalidad y estilos atribucionales de la población estudiada para este fin hemos tabulado y calculado los porcentajes para cada tipo de personalidad y estilo atribucional en función a la condición de empleo y desempleo cuyos resultados son los siguientes:

e.l Finalmente del total de examinados 483 sujetos, encontramos que el $73 \%$ de examinados pertenecen al tipo Productivo de Personalidad el 18\% son Apelativos 6\% Distanciadores y el $3 \%$ Destructivos. Respecto al estilo atribucional el $77 \%$ presentan un estilo atribucional optimista, en este grupo están el $50 \%$ de empleados, por otra parte el $23 \%$ del total presentan un estilo atribucional pesimista que incluye $15 \%$ de empleados y al $29 \%$ de desempleados, datos que pueden verse en la Tabla No. 6 .

e.2 Se observa que el porcentaje de sujetos con personalidad productiva y empleado es del $47.20 \%$ frente al $25.88 \%$ en el grupo de desempleados, cuya diferencia de 21.32 , con un error estándar de 3.04 arroja una razón crítica de diferencia de porcentajes de 7.01 que resulta muy significativa para el 0.01 de margen de error.

e.3 El $28.78 \%$ de los desempleados presentan un estilo atribucional pesimista en contraste al $14.90 \%$ de los empleados, la diferencia de 13.88 , con un error estándar de 4.01 tenemos una RC \% igual a 3.46, que resulta también significativa al 0.01 de riesgo de error.

e.4 De lo hallado se desprende que en el grupo de empleados predomina significativamente la personalidad productiva y el estilo atribucional optimista, tal como puede verse en la Tabla No. 6. 
Tabla Nro. 6

De frecuencias absolutas y relativas de los tipos de personalidad y estilos atribucionales en función a la condición de empleo y desempleo y para la muestra total

\begin{tabular}{|l|rr|rr|rr|}
\hline \multirow{2}{*}{ Pers. y atrib. } & \multicolumn{6}{|c|}{ Condición laboral } \\
\cline { 2 - 8 } & \multicolumn{2}{|c|}{ Empleados } & \multicolumn{2}{|c|}{ Desempleados } & \multicolumn{2}{|c|}{ Total } \\
\cline { 2 - 8 } & \multicolumn{1}{|c|}{$\mathbf{F}$} & \multicolumn{1}{|c|}{ F } & \multicolumn{1}{c}{ F } & \% \\
\hline Productivos & 228 & 47.2 & 125 & 25.88 & 353 & 73 \\
Apelativos & 56 & 11.59 & 31 & 6.42 & 87 & 18 \\
Distanciadores & 1 & 3.93 & 10 & 2.07 & 29 & 6 \\
Destructivos & 10 & 2.07 & 4 & 0.29 & 14 & 3 \\
\hline Total & $\mathbf{3 1 3}$ & $\mathbf{6 4 . 8}$ & $\mathbf{1 7 0}$ & $\mathbf{3 5 . 2}$ & $\mathbf{4 8 3}$ & $\mathbf{1 0 0}$ \\
\hline Estilo y Atrib. & & & & & & \\
Optimista & 241 & 49.9 & 131 & 27.12 & 372 & 77 \\
Estilo y Atrib. & & & & & & \\
Pesimista & 72 & 14.9 & 39 & 28.78 & 111 & 23 \\
Total & $\mathbf{3 1 3}$ & $\mathbf{3 4 . 8}$ & $\mathbf{1 7 0}$ & $\mathbf{3 5 . 2}$ & $\mathbf{4 8 3}$ & $\mathbf{1 0 0}$ \\
\hline
\end{tabular}

\section{DISCUSIÓN}

El estilo explicativo es una variable cognitiva de personalidad que refleja el modo en que las personas explican habitualmente las causas de sus fracasos, da cuenta de la falta de motivación o desmoralización ante situaciones que demandan logros (Peterson, 1990).

Si una persona tiende a atribuir las razones de sus fracasos o eventos negativos a causas internas, entonces estará minando su propia autoestima, sentido de valía personal y percepción de su capacidad para controlar las situaciones.

$\mathrm{Si}$ a ello se aúna la tendencia a pensar que las causas de los infortunios sufridos van a permanecer más allá del día de hoy y de la situación específica presente, tendremos que la persona estará atribuyendo su condición a factores estables y globales.

La conjunción de una explicación interna, estable y global, es la que mayor pesimismo y desmoralización producirá en el individuo. y es denominada por ello estilo explicativo pesimista.

Se ha encontrado que el estilo explicativo pesimista está asociado a la depresión e incluso a la pérdida de la salud físico (Peterson, 1992, 450), pero además de ellos Peterson cita los siguientes resultados como consecuentes de un estilo explicativo pesimista:

- Depresión (Sweeney, Anderson, \& Bailey, 1986)

- Bajas calificaciones en la escuela (Peterson \& Barret, 1987)

- Bajo desempeño laboral (Seligman \& Schulman, 1986)

- Soledad (Anderson, Horowitz, \& French, 1983)

- Mal estado de salud física (Peterson, Seligman \& Vaillant, 1988) 
Por su parte la personalidad desde el modelo de A. Adler en los complejo medio fin, se espera que el tipo productivo enfrente los problemas de la vida, contribuyendo siempre que sea necesario con el bienestar del entorno sin que para ello tenga que sacrificarse, con un enfoque diferente están los tipos apelativos quienes consiguen que sean quienes les resuelven su problemas. Los ti pos destructivos buscan resolver sus problemas mediante la fuerza y la agresividad. Los distanciadores no se esfuerzan por mejorar las cosas aún cuando lo perjudiquen, responden con evasivas. Como es de esperarse en la interacción humana estos cuatro tipos de personalidad, estarán presentes dándole matices claramente diferenciados comportamentalmente, al tiempo que cada quien también como característica de personalidad, estará enfrentando la vida cognitivamente, cuando utiliza los estilos explicativos de las cosas que le ocurren. De modo que debemos esperar que el tipo productivo sea también el optimista, que las dimensiones: internas, estables y globales que definen el compuesto negativo caractericen a los tipos improductivos. Los resultados confirman que cuanto más definido es el tipo productivo es también definidamente optimista y a la inversa cuando la personalidad es Distanciadora y Apelativa. No hay relación con la personalidad Destructiva pudiendo estos sujetos ser optimistas o pesimistas, esto se debería porque las personalidades de orientación destructiva son activos, enfrentan los problemas de la vida a diferencia de los Productivos, cargados de medios violentos.

Al analizar el tipo de personalidad en función a la condición de empleo y desempleo encontramos que solo hay diferencias en la personalidad productiva en dirección a los sujetos en condición de empleo. Por otro lado desde la concepción atribucional encontramos que la causa atribuida a uno mismo suele ser estable en el tiempo con tendencia a global izar definiendo un estilo pesimista de afronte que está en dirección a los sujetos en actual condición de desempleo en la muestra estudiada y $28.78 \%$.

De lo hallado se desprende que siendo el $73 \%$ de los examinados, elegidos aleatoriamente, sujetos con tipo de personalidad productiva y estando ésta relacionada con el estilo atribucional optimista estamos en condiciones de afirmar que significativamente en nuestro medio hay la clara tendencia para enfrentar y resolver los problemas, modificando las situaciones para que sean mejores y con buena capacidad para tomar decisiones por sí mismo.

\section{CONCLUSIONES}

1. Se acepta la hipótesis 1 , que nos dice que a más indicadores de personalidad productiva habrá menos indicadores de estilo atribucional pesimista, al encontrar un coeficiente de correlación de -0.35 que resulta significativa al 0.05 de margen de error.

2. Se acepta la hipótesis 2 que nos dice que la correlaciones entre el tipo de personalidad con el estilo atribucional difieren significativamente en función a la condición de empleo y desempleo en dirección al grupo de los empleados. Donde los sujetos en condición de empleo presentan mayor productividad y optimismo como lo confirma la prueba de diferencia de medias y de razón crítica de diferencia de porcentajes

3. Se rechaza la hipótesis 3 que nos dice que la correlaciones entre el tipo de personalidad con 
el estilo atribucional difieren significativamente en función al género, al no encontrar en ningún caso diferencias correlacionales.

4. En la muestra total el $73 \%$ de los examinados son del tipo de personalidad productiva y el $77 \%$ se caracterizan por afrontar la crisis con optimismo. Cuando la descripción se hace en función a la condicion de empleo encontramos que el $47 \%$ son de personalidad productiva y el $50 \%$ se caracterizan por tener un estilo atribucional optimista. Por otra parte solo, el $25 \%$ de los desempleados tienen una personalidad productiva y solo el $27 \%$ enfrentan la vida con optimismo.

\section{BIBLIOGRAFIA}

Allport G. (1965) Psicología de la Personalidad. Buenos Aires. Ed. Paidos.

Ad1er Alfred.( 1955) El Sentido de la Vida. Ed. Miracle.

Adler Al fred (1945) El Carácter Neurótico. Ed. Paidos.

Ansbacher Heinz y Ansbacher R. (1959) La Psicología Individual de Alfred Adler. B.Aires Ed. Troquel.

M. Titze. (1983)Fundamentos del teleonálisis adleriano. Barcelona. Ed. Herder.

OIT Informe sobre el empleo en el mundo 1998 - 1999. Papel Fundamental de la Formación.

M, Seligman Helplessness (1975) On depression, development and death. San Francisco. B.H Freeman.

Peterson y Villanova.( 1988) An expanded Attributional Style Questionnaire. Journal Of Abnormal Psychology, 91,87-89

Peterson, C. (1991) The Meaning an Measurement of Explanatory Style, In. Psychological Inquiry. Vol.2 Nro 1,1-10

Paredes Tarazana, Mildred, 1997, Tesis de Maestría "La Interdependencia Empresarial, Factor de Calidad, Sector Pesquero Peruano". UNMSM/FCA/Unidad de Post Grado. 